\title{
Alpina: Innovación permanente para "sorprender" al cliente
}

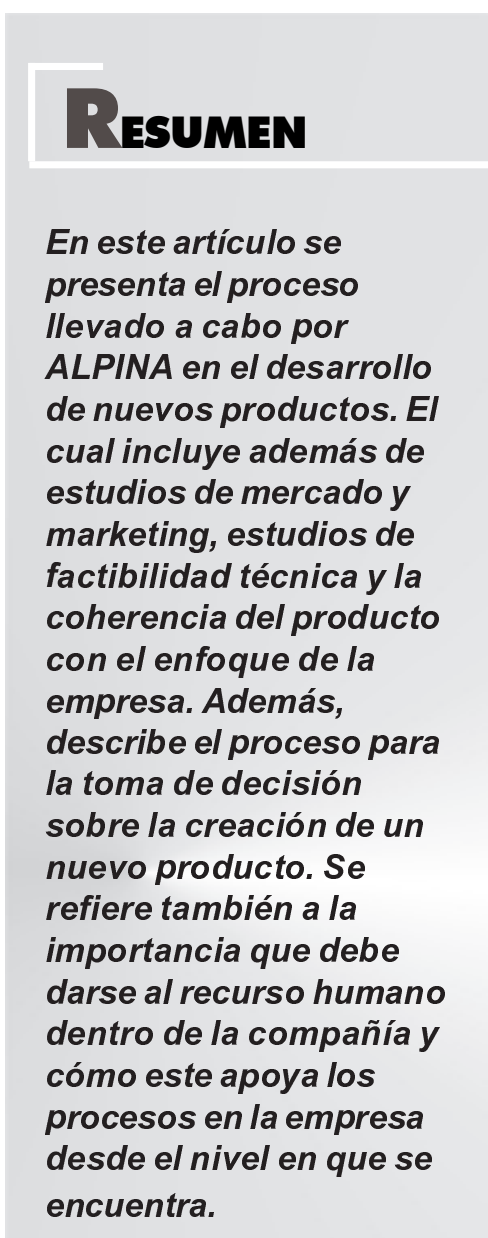

\section{Palabras clave}

Innovación, factibilidad técnica, nuevos productos, planeación estratégica
Por:

Mauricio Nieto Potes Gonzalo Castellanos ${ }^{1}$
$\mathbf{M}$

ás que descubrir productos novedosos o de tener permanentemente grandes ideas, lo que convierte a una empresa en una organización verdaderamente innovadora es su capacidad para desarrollar los procesos de gestión de las ideas y del conocimiento en el día a día de la organización, el contar con una cultura desarrollada que facilite la creación y el diseño de nuevos productos, nuevos procesos, mejoras e innovaciones en las tecnologías de gestión-gerencia y administración. En esta cultura desarrollada para la innovación caben también las innovaciones incrementales para la mejora de los "viejos" productos o los saltos innovadores para productos nuevos y exitosos no conocidos en el mercado.

Con casi 60 años de existencia en el mercado, ALPINA se ha constituido en una de las más importantes empresas de Colombia en el sector de alimentos y bebidas. Desde su creación y por voluntad de sus fundadores, ALPINA incorporó en su filosofía, en sus valores y en su accionar el principio orientador de la innovación. La fabricación en el país de tres tipos de queso

1 Ejecutivos entrevistados: Álvaro Otálora, Director Desarrollo tecnológico y María del Pilar Bolívar, Gerente coorporativo de comunicación organizacional de Alpina. Gonzalo Catellanos, investigador GPyMES-EAN/ Mauricio Nieto, Vicerrector Investigaciones-EAN. 
holandés y su aceptación por un mercado que para la época desconocía este producto dieron comienzo a un historial de innovaciones que ha sido permanente y que ha ofrecido a sus consumidores una gama de productos nuevos bajo la consigna de "sorprender al cliente"2. Como lo relata Álvaro Otálora Director de Desarrollo Tecnológico de Alpina:

Hacia el año 1960 se empezó a consolidar la empresa ALPINA, como una compañía láctea no como una compañía quesera; siendo una compañía familiar, comienza a desarrollar y a producir un yogurt hecho especialmente para el consumidor colombiano. En este momento el yogurt en Europa se conocía como un yogurt espeso; los dos dueños de nacionalidad suiza llegados al país en 1946 desarrollaron un yogurt relativamente líquido que es el llamado Yogurt ALPINA, que todavía se produce y que en aquel momento era una verdadera innovación. De alguna forma, ALPINA fue la responsable de incrementar el consumo per cápita de yogurten Colombia, a través de la diversificación del negocio.

Desde ese momento, en los inicios de la compañía, el fabricar productos que no existían en el mercado fue llevando a la organización a establecer una orientación innovadora, introduciendo de manera permanente mejoras y cambios importantes. Desde 1970 empieza a profesionalizar su gerencia, a ampliar el cubrimiento con canales comerciales, a instalar sedes o bodegas de distribución en distintas ciudades del país, cambios que requirieron casi 15 años y que mantuvieron y fortalecieron en ALPINA su vocación por la investigación y el desarrollo necesarios para el lanzamiento al mercado de nuevos productos. Se obligó así la compañía a adaptarse a las circunstancias cambiantes del entorno y a emplear colaboradores competentes y formados para el cambio, el liderazgo, el empoderamiento y el trabajo en equipo.

2

Nótese que este concepto de «sorprender al cliente» va más allá del concepto tradicional de «satisfacer las necesidades del cliente».

\section{A bstract}

This paper presents the process of new products development of Alpina.

This is a process that includes marketing and merchandising, technical feasibility and a

coherence analysis of each innovation with focus in the

organization. It is also described the process of

the decision making related to new products. It remarks on the human talent necessary and on how people support the whole process from every hierarchical level.

\section{KeY WORDS}

Innovations, technical feasibility, new products lunching, strategy planning. 


\section{Innovación y plan estratégico}

La innovación en ALPINA trasciende a toda la organización. Está presente en la Misión y en la Visión, en la declaración de principios y valores. Aparece unida al cliente o al consumidor que constituyen el punto de partida. Le ayuda a todo el personal a entender que sin importar en cuál lugar de la organización trabaja, en los "procesos operacionales o en los no operacionales", está claro que el punto de partida es el consumidor. No se depende de lo que haga mercadeo o de lo que piense o interprete el área técnica, sino que todo el personal está encaminado a responderle y a sorprender a esa persona que tiene las necesidades, que las ha manifestado o que sin manifestarlo espera ser sorprendido por productos nuevos. Por ello es que la "forma" elegida por ALPINA para responder a sus clientes, es la innovación.

A partir del conocimiento del cliente y de los principios rectores que inducen la innovación, ALPINA ejecuta un juicioso proceso de planeación estratégica. Han definido un ciclo de planeación que involucra el corto, mediano y largo plazo dentro de un marco de referencia que dicta la alta gerencia consistente en las orientaciones sobre nuevos negocios o en cambios que quiere introducir en el sector. El complemento es el manejo de la información y el uso de indicadores elaborados para el seguimiento de los procesos. ALPINA creó como parte de su espíritu innovador el "GEl" su propio sistema de seguimiento a la gestión.

Una vez la organización plantea los objetivos, estos se van formulando por niveles. Se definen iniciativas estratégicas que apuntan al mejoramiento del negocio y su ejecución se mide con indicadores de área que apuntan a unos indicadores de la compañía en general. Estos son monitoreados por el equipo directivo y sirven para evaluar la evolución de la empresa y para compararla con otras organizaciones.

\section{Del plan estratégico a las acciones concretas}

En ALPINA la planeación estratégica se vuelve realidad a través de la gestión estratégica individual. Este concepto lleva a cada persona que trabaja en ALPINA a preguntarse: ¿cómo aporto yo y en qué forma a los objetivos generales de la organización? ¿Cómo aportan mis colegas a los míos y los míos a los de mi jefe? Y así sucesivamente, hasta completar tres aspectos básicos que la organización monitorea: rentabilidad, participación en el mercado y crecimiento. Este proceso involucra además indicadores individuales de desempeño y planes de acción para empleados ubicados en procedimientos básicos: las personas que trabajan en la empresa saben que están siendo medidos en alguna forma específica, saben qué se espera de ellos y conocen qué objetivos precisos deben cumplir. Se ha diseñado y puesto en operación, además, un esquema de retroalimentación para que la gente sepa cuáles son los objetivos para los cuales la compañía está trabajando, cómo se van cumpliendo dichos objetivos, cuáles son los objetivos del siguiente año y todo esto dentro de una cadena de valor con diagramas precisos de flujo.

A partir del planteamiento de objetivos realizado por la organización, todos trabajan observando cómo pueden aportar a esos objetivos. Por ejemplo, ¿en qué objetivo de crecimiento puede aportarse? ¿Revisando el portafolio de productos o desarrollando nuevos productos o involucrándose en otros mercados? En este proceso todo el mundo 
participa con ideas y con proyectos en todos los niveles de la organización, hasta los más operativos. "La señora que apoya con el servicio de tintos aporta ideas de ahorro, economía y racionalización de recursos, lo que hace que ella esté contribuyendo efectivamente con los objetivos generales de la empresa."

Otro ejemplo de esta cascada de generación de ideas y compromisos que se va construyendo en los diferentes niveles de la empresa es el objetivo definido acerca del conocimiento del consumidor. Implica que se hagan proyectos de inteligencia y desarrollo de mercado y que en la parte del manejo operacional se haga referencia "al fortalecimiento de todas nuestras competencias industriales y logísticas para llegar al consumidor con calidad, con los precios y costos y con la oportunidad que se requiere".

En este campo del vector CPO -calidad, precio y oportunidad-ALPINA ha "desarrollado una serie de puntos que rigen básicamente este proceso y que orientan cómo mantener competitivo el negocio de nuestro portafolio actual, es decir, la ejecución de planes y programas para mantener los costos y la calidad dentro de los estándares que la empresa establece; también cómo desarrollar nuevos productos desde la óptica industrial, dentro del marco estratégico de la organización y cómo mantener y orientar el recurso humano para garantizar y mantener su competitividad".

Este último aspecto exige el desarrollo de competencias y un trabajo por parte del departamento de gestión del talento humano que define acciones de largo plazo incluyendo temas como el bilingüismo y las competencias en gestión y administración. Para lograr estas metas se trabaja en coordinación con algunas universidades estableciendo planes de desarrollo individual en los que se parte de evaluaciones de $360^{\circ}$ grados, para definirle a cada cargo un esquema de desarrollo por competencias. Estos son programas que se realizan en un horizonte de mediano y largo plazo.

\section{El lenguaje de la innovación en ALPINA}

En ALPINA la innovación ha estado "dentro de la sangre de la organización" y se la concibe bajo dos aspectos: el aspecto técnico y el organizacional. De un lado, que es el más comúnmente reconocido, la innovación se expresa en la introducción permanente de productos nuevos al mercado y llevó a que en 1982 la empresa conformara para el efecto un equipo de investigación y desarrollo -I\&Dcompuesto por dos personas una de las cuales fue un extranjero con altas calificaciones experto en leche quien organizó el departamento respectivo. El otro lado de la innovación se dio en ALPINA simultáneamente con las necesidades de crecimiento. Al respecto, comenta Maria del Pilar Bolívar, responsable de la Comunicación Organizacional en Alpina.

"Para que la innovación pueda tener buenos resultados se requiere tener en cuenta aspectos de la cultura, se necesita fortalecer los equipos de mercadeo con una conciencia muy clara hacia el consumidor y se deben tener competencias técnicas muy altas. Esa mezcla de elementos culturales, de trabajo en equipo $y$ de competencias técnicas, hacen que el surgimiento de innovaciones sea más frecuente $y$ de mayor impacto hacia el consumidor." 
En ALPINA se refieren a dos aristas de la innovación: "la operacional y la no operacional". Álvaro Otálora amplía estos conceptos que hacen parte del lenguaje de la empresa, comenzando por el concepto de innovación no operacional, entendiendo por ella un conjunto de acciones dirigidas a fortalecer la capacidad administrativa, el qué hacer con la gente, cómo pagarle, cómo remunerar el esfuerzo y el desempeño superior, cómo entender el negocio en un entorno complejo, cómo adaptarlo a los cambios del entorno incluido el tema de la modernización de la información, los temas de software, de la parte comercial hacia el cliente, de la adquisición de nuevas tecnologías y también la parte del manejo del conocimiento.

"El otro aspecto de la innovación es el operacional-señala Álvaro-, que se refiere básicamente al desarrollo de las habilidades técnicas de manufactura y de logística, para poder llegar al cliente al menor costo, más rápido y con una calidad por la cual él paga esas habilidades se desarrollan de forma paralela".

\section{Innovación y competencias del personal}

Hay otro aspecto diferencial en ALPINA y es que ha encontrado la forma de relacionar la innovación con las competencias. Puesto que hay diferentes niveles de desarrollo de estas competencias en los diferentes grupos de profesionales y empleados de la organización, se diseñan programas y acciones específicas para el desarrollo de determinadas competencias por áreas. Dentro de las competencias básicas de la organización por ejemplo- se identifican la innovación y la creatividad. En los grupos de trabajo de áreas como mercadeo y desarrollo tecnológico, algunos cargos administrativos y comerciales requieren que las competencias relacionadas con la innovación tengan niveles muy elevados de desarrollo, por ello para estas áreas se diseñan y realizan programas y actividades explícitas encaminadas a su fortalecimiento. Si dentro del plan de desarrollo personal se ve que la persona tiene falencias, se hace un plan de mejoramiento para llevarla a los niveles requeridos; luego que eso se ha logrado y comienza a formar parte de la cultura, y dado que se tiene claro para dónde se va dentro del marco de estratégico, se arranca un proceso, denominado proceso de innovación y desarrollo operacional. Se enmarcan allí cómo nacen las ideas, cómo se manejan y cómo se gerencian hasta al lanzamiento del producto.

\section{El proceso de innovación y el lanzamiento de productos}

Profundizando sobre el proceso de innovación y de desarrollo de productos, en ALPINA este es un proceso organizado, que tiene que ver con un plan de la compañía diseñado para el lanzamiento de productos. Este es un plan estructurado y claramente definido que se diseña en estrecha relación con el plan general de crecimiento. A manera de ejemplo, la organización define que el crecimiento del año fiscal debe ser del orden del $10 \%$ en ventas y en ese $10 \%$ se entiende que hay un crecimiento orgánico del portafolio existente, pero además que debe haber un crecimiento explicado por el desarrollo de nuevos productos. Los nuevos productos deben tener una cuota de innovación y una cuota de diferenciación, porque uno de los factores de éxito del mercado de este tipo de alimentos y bebidas es precisamente la diferenciación y el fortalecimiento de la compañía como una compañía innovadora. 


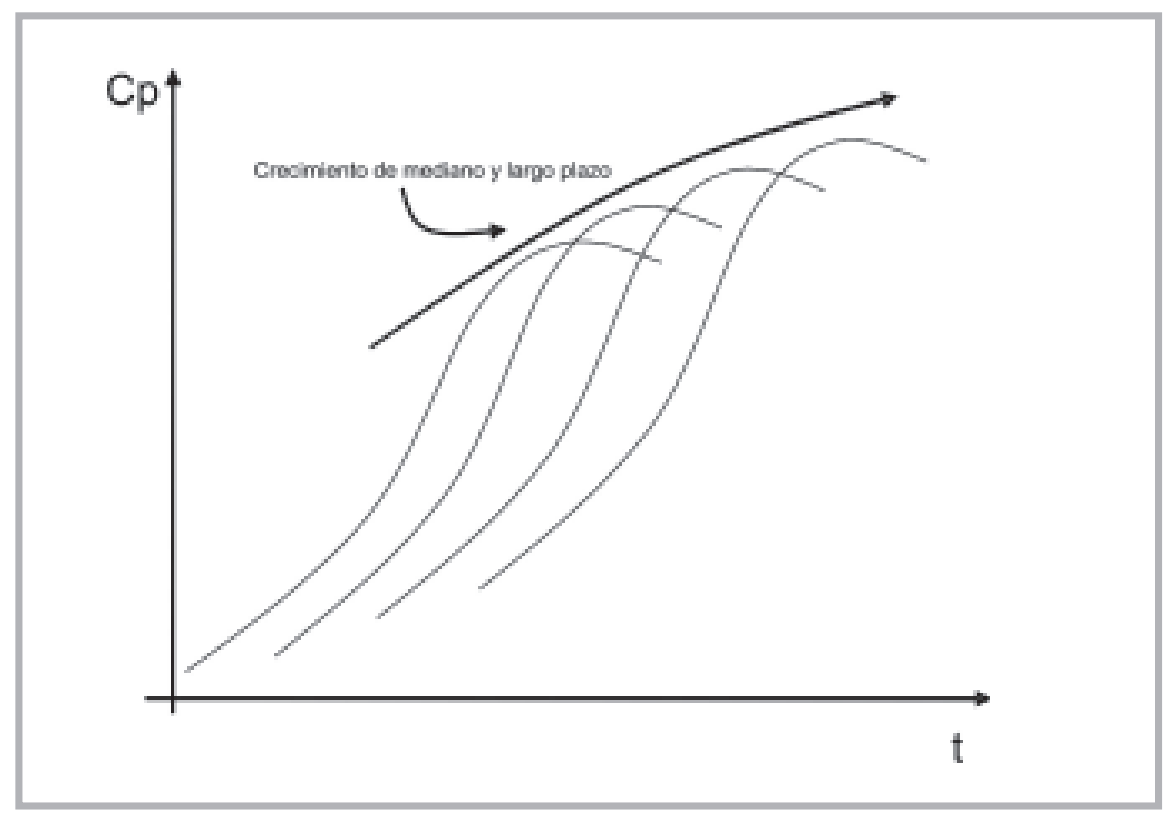

Como lo indica este gráfico, donde Cp es el ciclo de los productos a través del tiempo (t), la compañía basa su estrategia de crecimiento en el permanente lanzamiento de productos nuevos o en la mejoras de los productos viejos de forma que cuando un producto "viejo" va cumpliendo su ciclo y presenta síntomas de saturación y decrecimiento, productos mejorados o nuevos dan el recambio garantizando la curva de crecimiento para la organización en el mediano y el largo plazo, basada en la innovación.

En este aspecto está la clave del éxito de la compañía, en el reconocimiento de la innovación como factor explícito del compromiso de la compañía para dar una adecuada respuesta a las necesidades del consumidor, la conduce al crecimiento.

"Es importante señalar que ALPINA debió hacer un trabajo muy juicioso -señala Álvaro Otalora- para entender qué es la innovación aquí en Colombia o en la región y además qué es innovación en el mundo, porque entendemos también que este plan del negocio nos está llevando no solo a definir lo que necesita el mercado colombiano sino seguramente cómo las innovaciones de aquí van a ser también capitalizadas por otras regiones $u$ otros lugares donde estamos o vamos a estar presentes".

\section{Sobre la gestión de las ideas innovadoras}

Es clave retomar aquí el proceso de manejo de las ideas en ALPINA, que se había relacionado antes con el despliegue del plan estratégico y con las competencias de creatividad e innovación en el personal. Dentro del marco estratégico existen una serie de iniciativas que se traducen básicamente en números. Esto es un negocio y si se pretende crecer al $20 \%$ anual por ejemplo y el $50 \%$ de ese $20 \%$ debe ser explicado por los nuevos productos y de esos nuevos productos se debe lograr una cuota de innovación del $10 \%$ o del $15 \%$ lo cual se traduce en que por cada 100 productos lanzados se logran 2 ó 3 productos innovadores, "la innovación es una difícil cosecha". 
En ALPINA se están lanzando al mercado entre 20 y 25 productos por año, a nivel corporativo. ¿Cómo se maneja esto? Hay que señalar que en la actualidad no hay en la empresa un "banco de ideas" como lo puede haber en otras compañías que, por ejemplo, desarrollan software. Hasta hoy no se a creado una "gerencia de ideas" pero a corto plazo lo que sí se va a tener es un "banco de ideas". Con lo que se cuenta hoy es con un "banco de proyectos" y con procesos de gerencia de los proyectos que garantizan que las ideas que surgen en cualquier parte de la organización se conviertan en proyectos concretos. "La idea es que al levantarme hoy y abrir la nevera puede surgir una idea que rescatada y convertida en un proyecto puede resultar ser una gran idea". Las ideas se canalizan, porque es una responsabilidad de toda persona en la organización generar modificaciones e iniciativas; puede ser una persona de la planta, un gerente o un vendedor quien expone la idea, quien ve la oportunidad para mejorar: "oye que interesante, tener un bebida como la avena que venden en Cali".

La organización conoce cuales son los procesos y procedimientos para el manejo de una idea innovadora, cualquier empleado sabe a quien dirigirse para hablar de una idea. Los canales están definidos. Todos pertenecen a un equipo de trabajo, a equipos formales; todo operario participa en una reunión por mes donde se discuten las ideas y proyectos innovadores. Existen diferentes niveles o estructuras de equipos para la innovación. Los equipos primarios para áreas tan grandes como las de producción son equipos de trabajo donde el jefe se producción se reúne con su grupo o donde el jefe de mantenimiento se reúne con sus ayudantes. En todos estos grupos se están generando ideas.
Es inherente a la cultura innovadora y de mejoramiento continuo que "si yo identifico algo novedoso, lo digo. Es ahí donde se mezcla el tema de la comunicación abierta. Yo lo transmito porque es mi responsabilidad decirlo. Si trabajo con la expectativa de que me van a pagar la idea o lo comunico para que me lo reconozcan, no estoy viviendo en la cultura ALPINA". Las ideas se identifican y se capturan, porque todas las personas pasaron por una actividad de formación que se llama "innovación y creatividad" y se les entregaron una urna para depositar ideas y es parte de la responsabilidad de cada jefe si se llena o no; cada persona tiene dentro de su oficina una urna y coloca en esa urna lo que se le ocurra que puede ser una buena idea, puede ser una idea comercial, una idea administrativa o un nuevo producto. La innovación no está solamente en los laboratorios ni en reuniones periódicas de innovación; la creatividad se respira en la cultura de la organización.

¿Pero cómo hacen las personas para que sus ideas se conviertan en proyectos? Tiene que ver con cómo venden sus ideas a los diferentes niveles de la organización. "Hay muchos ejemplos de muchas cosas bellas que han surgido de los mismos operarios, de los mismos vendedores, y se han convertido en proyectos de mejoramiento de la organización o en productos estrella." Dentro de los grupos de trabajo existen comités especializados y dentro de estos se tienen uno de desarrollo de nuevos productos y otro de innovación, que incluye a los presidentes y vicepresidentes de la Compañía.

Se han conformado dos equipos que deben gerenciar el desarrollo del portafolio: uno para el tema de mercadeo y otro para desarrollo tecnológico encargado de llevar a la realidad las ideas y convertirlas en productos. 
El equipo de mercadeo orienta a los vendedores, respecto a las ideas de cada producto: actúa a modo de un auditor para ellos. Las ideas tienen que estar dentro del contexto de ALPINA, se puede decir que un salchichón es una buena idea pero se sale del foco de la empresa. ALPINA posee la plataforma tecnológica para saber si un producto es viable o no. Las ideas de cada proyecto son discutidas por los directores de mercadeo encargados de adelantar una crítica desde el punto de vista comercial.

Si un jugo puede o no entrar en el negocio que tiene ALPINA hoy, se decide teniendo en cuenta la parte técnica, representada en personas que deciden en ese mismo Comité de si se cuenta o no con la tecnología y si las personas que dieron la idea vieron realmente cosas que normalmente no se ven. En ese Comité de Desarrollo se aprueba la idea, como una opción, como una iniciativa. Cuando el Comité encuentra que la iniciativa es atractiva y viable, hay un grupo de proyecto, compuesto por la parte comercial, de mercadeo, por expertos en manejo de proyectos y por expertos en manejo de proyectos de infraestructura. Esos dos equipos trabajan en conjunto para que la idea viable se convierta en números y hechos y se pueda afirmar que es factible realizarla en un tiempo determinado, que las inversiones son de tal magnitud y que, comercialmente, mercadeo pueda decir: el volumen es de tal cantidad, se debe posicionar de tal forma, se pueden utilizar " $x$ " marcas, se deben realizar " $y$ " inversiones en publicidad. De esta manera, con el trabajo en equipo, se construye la idea y se la convierte en un proyecto y en una prefactibilidad general.

Si el negocio diseñado es entendido como un proyecto rentable se somete nuevamente al Comité de Desarrollo. El Comité dice: "estamos de acuerdo, no tenemos ninguna duda, hagan estas modificaciones", y lo sube al Comité de Innovación. A este comité de innovación no van todas las ideas, solo van las de real innovación. Los equipos de proyecto manejan 150 proyectos, el equipo de desarrollo maneja 50 que considera importantes y el equipo de innovación analiza únicamente 10: es un tamiz hacia la innovación real.

El Comité de Desarrollo también mira los productos derivados, los de mejora incremental. Existe un grupo de productos que se tienen que lanzar porque son cartas estratégicas para la organización, para moverse en un cambio de entorno comercial o en un cambio de entorno económico en cualquier mercado, en Venezuela, en Ecuador, en Colombia, en el mercado de Estados Unidos, en los mercados donde ALPINA tiene negocios. Se realiza un análisis para identificar esos productos derivados que no van a los Comités y que se utilizan en caso de que en el plan de desarrollo y de lanzamiento de nuevos productos falle, porque los productos no fueron exitosos comercialmente. Así siempre se tienen esos otros productos que garantizan el crecimiento y mantienen la curva de mediano y largo plazo en una pendiente positiva de crecimiento de la organización, todo ello basado en la innovación.

De esta forma, encontramos en ALPINA el caso de una organización realmente innovadora, una organización en la que las mejoras incrementales en los procesos de fabricación y en los productos, la identificación y el diseño de productos realmente novedosos -aquellos que sorprenden al consumidor-y las mejoras continuas en la gestión -gerencia y administración de la firma, se han convertido en parte del día a día y han permearon completamente la cultura de manera integral. ALPINA una firma innovadora que constituye un ejemplo a seguir para las organizaciones PyMES colombianas. 